\title{
Para-stomal Hernia in Para-rectus versus Trans-rectus Stomas: A Randomized Controlled Study
}

\author{
Mohamed K.F. Hamed, Amr H. Afifi \\ Department of General Surgery, Faculty of Medicine, Ain Shams University, Egypt
}

Background: Para-stomal hernia is a common complication following fecal diversion. It has been attributed to many factors and site of stoma creation in relation to the rectus abdominis muscle is believed to be one of them. This belief was recently debated. The aim of this research was to detect if the site of stoma creation whether through (trans-) or away from (para-) the rectus abdominis muscle has a role in the development of such a complication.

Methods: Sixty patients undergoing fecal diversion were included in a prospective controlled study. They were randomly allocated into one of two main groups; whether to undergo a para-rectus stoma creation or a trans-rectus stoma creation. All patients were followed-up for one year for the development of para-stomal hernia.

Results: There was no statistically significant difference between the two groups as regard to the incidence of developing para-stomal hernia.

Conclusion: Creating stomas away from the edge of the rectus abdominis muscle i.e. at a para-rectus site is not a risk factor for developing para-stomal hernia.

Key words: Para-rectus stoma, trans-rectus stoma, para-stomal hernia.

\section{Introduction}

A colostomy is a surgical procedure by which a stoma is constructed through exteriorization of the large intestine. A colostomy can be a loop colostomy or an end colostomy. A loop colostomy is defined as a stoma in which the entire loop of colon is exteriorized and both the proximal $\operatorname{limb}$ and the distal limb open into the common stoma opening i.e. both ends are not completely separated. An end colostomy is created from the proximal end of the colon; the distal end can be stapled or sewn shut and remains as a blind pouch, or it can be exteriorized separately. ${ }^{1}$

Among the various stoma complications, the parastomal hernia (PSH) is the most common. ${ }^{2}$ Goligher, even, went so far as to claim that some degree of parastomal herniation is inevitable given enough follow-up time. ${ }^{3}$

Parastomal hernia is broadly defined as an incisional hernia located at or immediately adjacent to a stoma. ${ }^{4}$

Due to the frequency of PSHs and the limited success rate of repair, attention has been focused on preventing PSHs at the outset when a stoma is fashioned. ${ }^{2}$

Both patient and operative technical factors have been implicated in the subsequent risk of parastomal hernia. $^{5}$

Individual patient characteristics that have been shown to be independent risk factors for PSH development include older age i.e. being more than $60,{ }^{6}$ increased BMI i.e. more than 30, respiratory comorbidity, diabetes mellitus and the presence of other abdominal wall hernias. ${ }^{7}$ Other factors in the literature that have been suggested but not validated include malnutrition, smoking status, chronic coughing, chronic constipation, ascites, corticosteroid use, and postoperative wound sepsis. ${ }^{4}$

Technical aspects related to ostomy creation have been suggested as risk factors for the development of parastomal hernias especially the site of stomal exit. ${ }^{8}$

The site of colostomy fashioning has been a matter of debate; while most of the authors prefer the colostomy to be brought through the lateral edge of the rectus abdominis muscle ${ }^{1,9}$ based mainly on a 130-patient study by Sjodahl et al. that demonstrated a significantly lower rate of parastomal hernia when the stoma was fashioned through a trans-rectus route as opposed to a lateral para-rectus approach (3 vs. $22 \%),{ }^{10}$ a recent Cochrane review in 2013 showed no statistically significant difference in the rate of parastomal hernia or stomal prolapse between the two techniques. ${ }^{11}$ As no clinical trials comparing the varying surgical techniques for ostomy creation exist, the ideal approach remains controversial. ${ }^{4}$

Creation of a colostomy alters the abdominal wall. Midline shift to the contralateral side of the colostomy due to decreased restraining forces at 
the site of the colostomy, and ipsilateral abdominal rectus muscle (ARM) atrophy distal to the level of the colostomy due to intercostal nerve damage may explain the increased rate of parastomal hernias following the trans-rectus approach. ${ }^{12}$

Lacking enough evidence to be a risk factor for para-stomal hernia development, fashioning stomas at a para-rectus site appears to be an appealing option especially in cases of emergency surgery and cases where there is marked adhesions preventing colon dissection to reach the trans-rectus site freely.

\section{Methods}

After taking the approval of "The ethical committee for scientific research" regarding the sample size and the ethical points, sixty (60) patients presenting at our hospital (Ain Shams University Hospitals) who were decided to have temporary sigmoid colostomy as a mean of fecal diversion due to different indications, were included in this prospective study provided that they met our inclusion criteria: being within ASA I/II grade and with anterior abdominal wall muscles of accepted integrity and power (muscles were of intact integrity and motor power i.e. not denervated, lacerated nor paralyzed). Patients with generalized peritonitis were excluded except if the patient presented and was operated upon within 24 hours of the symptoms development (Those patients usually have a thickened mesentery requiring a wide aperture in the abdominal wall for stoma creation making those individuals more liable to develop para-stomal hernia especially after recovery).

Having obtained an informed consent from them or first-degree relatives if not possible, patients were allocated randomly in one of the two groups: group (A) representing patients with para-rectus stomas and group (B) representing those with trans-rectus ones. Each group was further subdivided into: subgroup (1) with end stomas and sub-group (2) with loop stomas

A thorough history was taken from each patient with special concern about the indication of diversion, associated co-morbidities and other individual patient characteristics that have been shown to be independent risk factors for PSH development include older age (more than 60), increased BMI (more than 30), respiratory comorbidities, diabetes mellitus and the presence of other abdominal wall hernias.

After completion of the previously decided surgical intervention, the stoma site was incised at about 3-5 $\mathrm{cm}$ from the lateral edge of the rectus abdominis muscle i.e. at a para-rectus position (in group A) or at the lateral edge of the rectus abdominis muscle i.e. trans-rectus (in group B). The stoma was then brought out from an aperture about $3 \mathrm{~cm}$ and was matured using full thickness vicryl 2/0 sutures (the aperture was about $4 \mathrm{~cm}$ in case of loop stomas). It is to be mentioned that patient allocation in either groups was done using the random computerization method and most of the pre-assumed risk factors for PSH were taken in consideration while allocating patients within the two groups; age "with the age of sixty as the cut-off point", co-morbidities affecting tissue perfusion and wound healing "especially DM and ischemic heart disease", obesity "taking the BMI value 30 as the cut-off point" and causes of persistent straining "mainly physical occupational stress, chronic constipation, smoking, respiratory co-morbidities, previous abdominal exploration, advanced intra-abdominal malignancies and other causes of persistent increase in the intra-abdominal pressure".

Patients were followed up regularly on monthly basis after being discharged for one year (or till the reversal of colostomies) as regards the position of the stoma and the presence of parastomal hernia clinically. Scanning Pelvi-abdominal CT was done for selected cases whose diagnosis of PSH was query despite being symptomatic (frequent colics and unexplained vomiting with normal serum electrolytes) and could stand being exposed to high dose of radiation and IV contrast during that study.

The results were reported in line with Consolidated Standards of Reporting Trials (CONSORT) Guidelines.

It is to be mentioned that interim analysis of the outcome obtained in the first five (5) then ten (10) patients was done by the ethical committee taking the approval to continue our study for the whole sample size.

\section{Results}

In this study, the incidence of developing PSH was assessed in sixty patients who underwent fecal diversion for various indications after a period of oneyear follow-up. All patients included, were recruited starting from January 2017 till January 2018 with the last patient in the study being followed-up till January 2019.

The primary endpoint of this study was the development of PSH within a time interval of six months.

All the data obtained was analyzed using the SPSS program.

The study included 24 male patients (40\%) and 36 females (60\%).

Forty-Eight (48) patients (80\%) were below the age of sixty with 36 patients of the whole study group having a BMI below or equal to 30 i.e. non-obese. Fourteen (14) patients were diabetics (Table 1). 
Causes of continuous straining were analyzed in the whole sample group and the results were as shown in Table 2.

The indications for fecal diversion were studied as shown in Table 3.

We had 32 patients in the para-rectus stoma group (Group A); 24 patients of them were in the end stoma group (Group A1) and 8 of them were in the loop stoma group (Group A2), while 28 of the patients were in the trans-rectus stoma group (Group B); 14 patients had an end colostomy (Group B1) and 14 were in the "loop" group (Group B2) (Table 4).

We had 3 cases that developed para-stomal hernias (PSH). The data of those cases were re-analyzed separately and each case was given a specific Latin number (I, II, III) (Table 5).

Table 1:Demographic data of patients

\begin{tabular}{|c|c|c|c|c|}
\hline \multicolumn{5}{|l|}{ Variable } \\
\hline \multirow[t]{3}{*}{ Gender } & Male & & Female & \\
\hline & $24(40 \%)$ & & $36(60 \%)$ & \\
\hline & Para $=13$ & Trans $=11$ & Para $=19$ & Trans $=17$ \\
\hline \multirow[t]{3}{*}{ Age group } & Young (be & & Old (60 or & \\
\hline & $48(80 \%)$ & & $12(20 \%)$ & \\
\hline & Para $=28$ & Trans $=20$ & Para $=4$ & Trans $=8$ \\
\hline \multirow[t]{3}{*}{ Obesity } & \multicolumn{2}{|c|}{ Non-obese (BMI below 30) } & \multicolumn{2}{|c|}{ Obese (BMI 30 or above) } \\
\hline & \multicolumn{2}{|l|}{$36(60 \%)$} & \multicolumn{2}{|l|}{$24(40 \%)$} \\
\hline & Para $=24$ & Trans $=12$ & Para $=8$ & Trans $=16$ \\
\hline
\end{tabular}

Table 2: causes of continuous straining

\begin{tabular}{|c|c|c|}
\hline Occupations with physical stress (manual workers and house-wives) & Yes & No \\
\hline & $36(60 \%)$ & $24(40 \%)$ \\
\hline \multirow[t]{2}{*}{ Chronic constipation } & Yes & No \\
\hline & $30(50 \%)$ & $30(50 \%)$ \\
\hline \multirow[t]{2}{*}{ Smokers } & Yes & No \\
\hline & $26(43.3 \%)$ & $34(56.7 \%)$ \\
\hline \multirow[t]{2}{*}{ Respiratory co-morbidities } & Yes & No \\
\hline & $10(16.7 \%)$ & $50(83.3 \%)$ \\
\hline \multirow[t]{2}{*}{ Previous explorations } & Yes & No \\
\hline & $4(6.7 \%)$ & $56(93.3 \%)$ \\
\hline \multirow[t]{2}{*}{ Advanced malignancy } & Yes & No \\
\hline & $16(26.7 \%)$ & $44(73.3 \%)$ \\
\hline \multirow{2}{*}{$\begin{array}{l}\text { Other causes of persistent increase in the intra-abdominal pressure } \\
\text { (ascites, peritoneal dialysis.......... etc.) }\end{array}$} & Yes & No \\
\hline & $15(25 \%)$ & $45(75 \%)$ \\
\hline Total $(n)$ & \multicolumn{2}{|c|}{$41(68.3 \%)$} \\
\hline
\end{tabular}


Table 3: Indications of diversion

\begin{tabular}{lccccccc}
\hline $\begin{array}{l}\text { Indication for } \\
\text { Diversion }\end{array}$ & Malignancy & $\begin{array}{c}\text { Bleeding per } \\
\text { rectum }\end{array}$ & MVO & Volvulus & $\begin{array}{c}\text { Iatrogenic colon } \\
\text { injury }\end{array}$ & $\begin{array}{c}\text { Frozen abdomen } \\
\text { / pelvis }\end{array}$ & $\begin{array}{c}\text { Pelvic / } \\
\text { perineal } \\
\text { sepsis }\end{array}$ \\
\hline $\begin{array}{l}\text { Number } \\
\text { (percentage) }\end{array}$ & 18 & 2 & 4 & 10 & 8 & 6 & 12 \\
\hline
\end{tabular}

Table 4: Segregation of the study group

\begin{tabular}{|c|c|c|c|c|}
\hline \multirow[t]{2}{*}{ Group } & \multicolumn{2}{|c|}{ Para-rectus group (A) } & \multicolumn{2}{|c|}{ Trans-rectus group (B) } \\
\hline & End (A1) & Loop (A2) & End (B1) & Loop (B2) \\
\hline & $24(40 \%)$ & $8(13.3 \%)$ & $14(23.3 \%)$ & $14(23.3 \%)$ \\
\hline Total (n) & \multicolumn{2}{|c|}{32} & \multicolumn{2}{|c|}{28} \\
\hline
\end{tabular}

Table 5: Patients with PSH

\begin{tabular}{lccccccc}
\hline & group & age & BMI & Co-morbidities & Indication for diversion & $\begin{array}{c}\text { straining } \\
\text { Interval for hernia } \\
\text { development }\end{array}$ \\
\hline Case I & A1 & 68 & 29 & $\begin{array}{l}\text { Ischemic heart dis- } \\
\text { ease (MI, E.F. } 35)\end{array}$ & $\begin{array}{c}\text { Perforated diverticular disease } \\
\text { Mesh erosion of colon after }\end{array}$ & yes & 3 months \\
$\begin{array}{l}\text { Case } \\
\text { II }\end{array}$ & A2 & 62 & 36 & HTN, asthmatic & $\begin{array}{c}\text { recurrent para-umbilical hernia } \\
\text { repair }\end{array}$ & yes & 2 months \\
$\begin{array}{l}\text { Case } \\
\text { III }\end{array}$ & B2 & 63 & 32 & DM, HTN, Asthmatic & $\begin{array}{c}\text { Perineal sepsis (Fornier gan- } \\
\text { grene }\end{array}$ & yes & 5 months \\
\hline
\end{tabular}

\section{Discussion}

Para-stomal hernia is a common complication following fecal diversion. ${ }^{13}$ Parastomal hernias develop in $4 \%-48 \%$ of colostomies and $1.8 \%-28 \%$ of ileostomies. ${ }^{6}$ About $30 \%$ of patients with $\mathrm{PSH}$ require surgical intervention, most commonly due to discomfort, poor fit of the ostomy appliance, or rarely due to obstruction, bowel perforation, or strangulation. ${ }^{14}$ Successful repair of a parastomal hernia can be challenging. ${ }^{15}$ Due to the frequency of PSHs and the limited success rate of repair, attention has been focused on preventing PSHs at the outset when a stoma is fashioned. ${ }^{2}$ The clinical diagnosis of a parastomal hernia is reasonable accurate, with the majority of parastomal hernias occurring in the first year. ${ }^{15}$

The site of stoma creation in relation to the rectus abdominis muscle has been classically considered to be one of the factors responsible for the development of PSH after faecal diversion. ${ }^{10}$ To create the stoma at the lateral edge of the rectus abdominis muscle has been "the standard of care" until this was recently debated. ${ }^{11,13}$

In this study, we assessed sixty (60) patients undergoing faecal diversion for different indications and the incidence of PSH occurrence was determined after a period of one-year follow-up to determine the role of that factor i.e. the relation of the stoma to the rectus abdominis muscle, in hernia development.

Throughout the study, we standardized the whole process; whether the surgical operation being done by the same surgical team, adopting the fast tract recovery protocol, giving the same postdischarge instructions, having the same frequency of post-discharge visits or being followed-up by the members of the surgical team.

Clinical examination was adopted as a reliable mean for detecting the occurrence of PSH in face of using the pelvi-abdominal computed tomography (PACT) with contrast. This was attributed to many reasons. It is convenient to all patients allowing standardization of the mean of PSH detection and it is cost-effective. Not all the patients can tolerate being given intravenous and oral contrast nor can withstand being exposed to a high dose of radiation during the PACT study especially those with advanced malignancy. This goes in accordance with the study done by Gil et al. who proposed a classification for parastomal hernias based on physical examination of the patient. ${ }^{16}$ Moreno-Matias in 200917 and Seo in 
2011,18 however, used the computed tomography imaging to classify PSH in another way. Gil et al. in 2011 classified PSH into four types: Type I representing isolated, small parastomal hernia, Type II representing small parastomal hernia with coexisting midline incisional hernia without any significant front abdominal wall deformity, type III representing isolated, large parastomal hernia with front abdominal wall deformity and Type IV representing large parastomal hernia with coexisting midline incisional hernia, with front abdominal wall deformity. Since it relied on physical examination of the patient and included suggestions of suitable surgical treatment for the respective types of hernia, it was the most clinically usable classification ${ }^{19}$ and clinical examination is sufficient for the diagnosis of parastomal hernia. ${ }^{13}$ To avoid missing patients during the clinical examination, patient should be examined in both the supine and erect positions sometimes while doing Valsalva maneuver. The ostomy appliance should be removed. It is also important to palpate the fascial edges through the intestinal wall.

Analyzing the results of the patients included in this study, we found that the site of stoma creation in relation to the rectus abdominis muscle is not a risk factor for the development of PSH; whether it is a loop or an end colostomy. We had three patients who developed PSH for whom further analysis was done. They were distributed in three of the four sub-groups; A1, A2 and B2. However, they were all old-aged patients with serious co-morbidities and ambient sepsis that would essentially impair wound healing and increase straining in the post-operative period in such frail patients: all of the three patients were above 60 years, one of them was suffering from ischemic heart disease with myocardial infarction and the other two were suffering from chronic respiratory problems. Similar results were concluded by many authors in many retrospective studies. Williams et al. ${ }^{20}$ reviewed 46 patients having colectomy with ileostomy for inflammatory bowel disease; 33 with ulcerative colitis and 13 with Crohn's disease. They identified PSH in 37\% of patients with "trans-rectus" stomas (6 of 16 patients) in face of $33 \%$ of those with "para-rectus" stomas (4 of 12 patients). Carne et al..$^{21}$ reviewed 6 studies retrospectively about the effect of stoma site. 344 with "trans-rectus" stoma and 167 at the "para-rectus position. They all found no significant difference in the incidence of PSH development with the except of 1 study done by Sjodahl et al. ${ }^{10}$ on 130 patients.

Patients in our study were followed-up for a period of one year. This was a satisfactory period for analysis of data on the short term; as most cases occurs in the first year 15 despite the fact that "PSH can appear as late as 20 years after the initial surgery" 22

The idea to create the stoma in a site that is not confined to the edge of the rectus abdominis muscle can be very beneficial even it may be the only available option in some situations such as cases with multiple previous operations leading to the well-known state of "frozen abdomen". It has another advantage of allowing the creation of stoma with minimal dissection of the mesentery.

A "para-rectus" colostomy may even decrease the incidence of the development of midline incisional hernia decreasing the incidence of abdominal rectus muscle (ARM) atrophy helping the identification and easy dissection of sub-costal nerve and other abdominal wall innervation because being lateral to the ARM, they are still less segmented. ${ }^{12}$

\section{Conclusion}

Creating colostomies or stomas in general away from the lateral edge of the rectus abdominis muscle i.e. at a "para-rectus" position is not associated with an increased risk of having para-stomal hernia and is as effective as, if not better, creating those stomas at the "trans-rectus" site.

\section{Limitations}

Being a self-funded study incorporating patients with various co-morbidities, it was not easy to include an objective CT study with contrast for the abdominal wall to fully study the effect of creating stomas at both of the proposed sites. We believe that would give rise to many points helping to adequately chose patients fit for which of these types of stomas i.e. whether having a "para-rectus" or a "trans-rectus" stoma.

\section{Disclosure and Conflict of interest}

The authors have no disclosure and there is no conflict of interest.

\section{References}

1. Vijayasekaran A, Tsikitis VL: Loop Colostomy: Medscape website. Available at: https://emedicine.medscape.com/article/1892803overview. Visited on: 2018.

2. Sohn YJ, Moon SM, Shin US, Jee SH: Incidence and risk factors of parastomal hernia. $\boldsymbol{J}$ Korean Soc Coloproctol. 2012; 28(5): 241-246.

3. Szczepkowski M, Gil G, Kobus A: Parastomal hernia repair--Bielañski Hospital experience. Acta Chir Iugosl. 2006; 53(2): 99-102.

4. Aquina $\mathrm{CT}$, Iannuzzi JC, Probst $\mathrm{CP}$, Kelly $\mathrm{KN}$, Noyes K, Fleming FJ, et al: Parastomal hernia: A growing problem with new solutions; Review 
Article; Dig Surg. 2014; 31: 366-376.

5. Funahashi $K$, Suzuki $T$, Nagashima $Y$, Matsuda S, Koike J, Shiokawa $H$, Ushigome $M$, et al: Risk factors for parastomal hernia in Japanese patients with permanent colostomy. Surg Today. 2014; 44: 1465-1469.

6. Pilgrim $\mathrm{CH}$, McIntyre $\mathrm{R}$, Bailey $\mathrm{M}$ : Prospective audit of parastomal hernia: Prevalence and associated comorbidities. Dis Colon Rectum. 2010; 53: 71-76.

7. Nastro $P$, Knowles $\mathrm{CH}$, McGrath $\mathrm{A}$, Heyman B, Porrett TR, Lunniss PJ: Complications of intestinal stomas. Br J Surg. 2010; 97: 18851889.

8. Hong SY, Oh SY, Lee JH, Kim DY, Suh KW: Risk factors for parastomal hernia: Based on radiological definition. J Korean Surg Soc. 2013; 84: 43-47.

9. Novell R, Li MKW, Ogunbiyi O, Cheung HYS: Transverse and sigmoid colostomies in colon in Kirk's general surgical operations, sixth edition. 2013; 230: 232, Elsevier.

10. Sjodahl R, Anderberg B, Bolin T: Parastomal hernia in relation to site of the abdominal stoma. Br J Surg. 1988; 75: 339-341.

11. Hardt J, Meerpohl JJ, Metzendorf MI, Kienle P, Post S, Herrle F: Lateral pararectal versus transrectal stoma placement for prevention of parastomal herniation. Cochrane Database Syst Rev. 2013. 11:CD009487.

12. Timmermans $L$, Deerenberg $E B$, van Dijk SM, Lamme $B$, Koning $A H$, Kleinrensink GJ, et al: Abdominal rectus muscle atrophy and midline shift after colostomy creation. Surgery. 2014; 155(4): 696-701.

13. Harb WJ: Reprint of Parastomal hernia surgical management. Seminars in Colon and Rectal Surgery. 2018; 29: 194-198.
14. Donahue TF, Bochner BH, Sfakianos JP, Kent M, Bernstein M, Hilton WM: Risk Factors for the Development of Parastomal Hernia after Radical Cystectomy. J Urol. 2014; 191(6): 1708-1713.

15. Fitzgerald MJ, Ullrich S, Singh K, Misholy O, Kingham P, Brady MS: Parastomal hernia repair using the "top hat" technique - An initial experience in 30 patients at Memorial Sloan Kettering Cancer Center. The American Journal of Surgery. 2018; 216: 465-470.

16. Gil G, Szczepkowski M: A new classification of parastomal hernias from the experience at Bielanski Hospital in Warsaw. Pol Przegl Chir. 2011; 83: 430-437.

17. Moreno-Matias J, Serra-Aracil $X$, Darnell-Martin A, Bombardo-Junca J, Mora-Lopez L, AlcantaraMoral $M$, et al: The prevalence of parastomal hernia after formation of an end colostomy. A new clinico-radiological classification. Color Dis. 2009; 11: 173-177.

18. Seo SH, Kim HJ, Oh SY, Lee JH, Suh KW: Computed tomography classification for parastomal hernia. J Korean Surg Soc. 2011; 81: 111-114.

19. Styliński R, Alzubedi A, Rudzki S: Parastomal hernia - current knowledge and treatment. Videosurgery Miniinv. 2018; 13 (1): 1-8.

20. Williams JG, Etherington R, Hayward MW, Hughes LE: Paraileostomy hernia: A clinical and radiological study. Br J Surg. 1990; 77: 13551357.

21. Carne PW, Robertson GM, Frizelle FA: Parastomal hernia. Br J Surg. 2003; 90: 784-793.

22. Gurmu A, Matthiessen $P$, Nilsson $S$, Påhlman $L$, Rutegård J, Gunnarsson U: The inter-observer reliability is very low at clinical examination of parastomal hernia. Int J Colorectal Dis. 2011; 26: 89-95. 